\title{
A Diffusion Tensor Imaging study to compare normative fractional anisotropy values at the lumbar spine with patients suffering from low back pain
}

\section{Kaushik Nayak}

SRINIVAS UNIVERSITY

Dr. Lakshmikanth H K

MANIPAL ACADEMY OF HIGHER EDUCATION, Manipal

Dr. Rahul P Kotian ( $\square$ kotian.rahul18@gmail.com)

Assam Downtown University https://orcid.org/0000-0003-2682-158X

Sushil Yadav

Manipal Academy of Higher Education, Manipal

\section{Research Article}

Keywords: fractional anisotropy, diffusion tensor imaging, low backache, neuroimaging

Posted Date: April 15th, 2021

DOI: https://doi.org/10.21203/rs.3.rs-424817/v1

License: (c) (i) This work is licensed under a Creative Commons Attribution 4.0 International License.

Read Full License 


\section{Abstract}

Background: Backache radiating to the limbs is the most common cause for nerve root compression which can lead to several functional abnormalities. Magnetic resonance imaging (MRI) provides valuable information regarding the size, shape, location and impact of disc herniation on the nerve root fibres, so MRI is used in the diagnosis of the disc herniation but inconsistencies were seen between the clinical symptoms and MRI findings. Diffusion tensor imaging (DTI) of lumbar nerve root fibres reduces the number of false positive and false negative findings and thus increases the concordance between clinical and imaging findings. Fractional anisotropy (FA) values ranges from 0 and 1 which describes the degree of anisotropy of a diffusion process. The current study explored the difference in FA values between subjects suffering from with and without low back pain using DTI.

Materials and Methods: 45 patients with low backache and 45 controls were employed to evaluate changes of the lumbar spine using DTI derived FA values. A Philips Achieva 1.5 Tesla MRI unit was used for data collection. In the present study we evaluated both FA and Apparent Diffusion Co-efficient (ADC) values in six regions of the lumbar spine.

Results: Patients with low backache showed differences in FA values compared to control group. Descriptive data analysis was done using SPSS software version 20.0. However the values did not follow normal distribution so Mann Whitney $U$ test was used to correlate FA and ADC values in both the groups. Significant differences were found in FA at both sides of the L4 vertebrae. We also found significant differences in $A D C$ at the left side of $L 3$ and right side of the $L 4$ vertebrae.

Conclusion: : Our findings indicate that DTI and FA can be used in patients with low backache for early detection and treatment. The affected side of the spine showing symptoms of the nerve roots showed significant reduction in FA and slight increase in ADC values.

\section{Introduction}

Lumbar disc herniation with radiculopathy is one of the most common disease caused due to nerve root compression which can lead to several functional abnormalities. Radiculopathy with nerve root bothering is experienced in degenerative lumbar spine sickness, most usually because of spondylosis or plate herniation. Anatomical MRI utilizing two-dimensional T1 and T2 weighted imaging exhibits intervertebral plate or neural foramen which are the main causes of nerve root compression found in subjects without manifestations making it hard to recognize a particular pathology ${ }^{(1)}$. Routine MRI is not able to provide quantitative information regarding pre and post-operative nerve root damage, hence DTI is used as a qualitative tool for identification of nerve roots paths which are not readily observed by conventional MRI. DTI can be used to visualize the nerve fibres non-invasively and has been widely used for reviewing of intense spinal canal injury and pre-operative restriction of any lesions ${ }^{(2)}$.

DTI additionally gives data about measure and careful area of circle herniation and its impact on the nearby nerve strands. DTI is the main technique which can give the backhanded perspective on the 
microstructure of the sensory tissue; not-withstanding the pathway of the fibres ${ }^{(1)}$. DTI with fibre tractography has discovered clinical applications in assessing any focal sensory system and has been widely used in imaging of nerve fibre tract ${ }^{(3)}$. This strategy investigates the anisotropic infinitesimal brownian movement of water particles along the particular direction of the anxious filaments. It can likewise be utilized in the analysis of numerous sclerosis and myelopathy because of cervical spondylosis. DTI permits the calculation of eigen values in each voxel, which are utilized to calculate the FA and MD (2). DTI of lumbar nerve root filaments decreases the quantity of false positive and negative discoveries and in this way builds the concordance among clinical and imaging findings ${ }^{(4)}$. DTI and tractography can be used for depicting the 3D structure of nerve roots and following changes of dispersion water particle inside the tissue and giving a gauge of free dispersion if unbound water.

\section{Methods}

\subsection{Subjects}

This was a case-control study with a retrospective design. A total of 45 patients with low backpain aged 18-55 years ( 25 males, 20 females, mean age: $64.05 \pm 8.79$ years); and 45 healthy control subjects ( 25 males, 20 females, mean age: $65.05 \pm 8.37$ years) were enrolled and included in the study. Patients suffering from disc herniation and compression were excluded from the study.

\subsection{Image Acquisition}

Images were acquired on a 1.5-Tesla Philips Achieva, class IIA series using a HNS coil system. DTI images were acquired using a single shot echo-planar imaging (EPI) sequence with the following parameters: b-value $=800, \mathrm{TR} / \mathrm{TE} /$ slice thickness $=4400 \mathrm{~ms} / \mathrm{ms} / 2 \mathrm{~mm}$, matrix size $=112 \times 110$, and FOV $=160 \times 160 \mathrm{~mm}$. Parallel imaging was used, with an acceleration factor of two and generalized auto calibrating partially parallel acquisition (GRAPPA) reconstruction and EPI factor of 55. Diffusionweighting gradients were applied in 15 non-collinear directions.

\subsection{Image Analysis \& Post-processing}

ROI identification and estimation of FA values were done using the Philips extended workspace. The DTI image sets were loaded along with sequence VISTA for anatomy correlation to visualize and calculate FA values. Sagittal section of DTI and VISTA sequence was taken. The original DTI and VISTA sequence obtained were sent to Philips Extended Workstation and the DTI sequence was superimposed with VISTA sequence.

\subsection{DTI derived measures from ROls}

A ROI technique was used to estimate FA values. ROI's using standard techniques and anatomy reference overlap sequences were drawn in the lumbar spine regions using DTI Philips extended MR fiber track 
software version 7.1.5.1. A 6 voxel ROI was placed on both right and left side of L3, L4 and L5 lumbar vertebrae in sagittal section and FA and ADC values were obtained as depicted in figure 1.

\subsection{Statistical Analysis}

The descriptive data obtained was analysed using SPSS version 20.0 and mean FA and ADC values were calculated. The values did not follow the normal distribution so Mann Whitney U-test was done to see the correlation between FA and ADC values in individuals with or without lower back pain.

\section{Results}

\begin{tabular}{|c|c|c|c|c|c|}
\hline Lumbar Vertebrae & SIDE & DTI VARIABLES & CASES & CONTROLS & p-value \\
\hline \multirow[t]{4}{*}{ L3 } & \multirow[t]{2}{*}{ RIGHT } & FA & 0.7664 & 0.7968 & 0.0304 \\
\hline & & $A D C$ & 0.2252 & 0.1887 & 0.0365 \\
\hline & \multirow[t]{2}{*}{ LEFT } & FA & 0.7948 & 0.7476 & 0.0472 \\
\hline & & $A D C$ & 0.1661 & 0.2524 & 0.8683 \\
\hline \multirow[t]{4}{*}{ L4 } & \multirow[t]{2}{*}{ RIGHT } & FA & 0.6569 & 0.7797 & 0.1218 \\
\hline & & $A D C$ & 0.2888 & 0.2209 & 0.0671 \\
\hline & \multirow[t]{2}{*}{ LEFT } & FA & 0.7272 & 0.8227 & 0.0955 \\
\hline & & $A D C$ & 0.2190 & 0.1695 & 0.0495 \\
\hline \multirow[t]{4}{*}{ L5 } & \multirow[t]{2}{*}{ RIGHT } & FA & 0.7144 & 0.762 & 0.0476 \\
\hline & & $A D C$ & 0.2153 & 0.2141 & 0.0012 \\
\hline & \multirow[t]{2}{*}{ LEFT } & FA & 0.7523 & 0.7923 & 0.0406 \\
\hline & & $A D C$ & 0.1928 & 0.2049 & 0.0121 \\
\hline
\end{tabular}

The results showed that difference was found in ADC values in left side of $L 3$ vertebrae and a significant changes in FA values were noted in both side of $L 4$ vertebrae. There was also a change in ADC values in the right side of the $L 4$ vertebrae. There was no significant difference found in FA and ADC values in other nerve roots when compared with individuals with back pain to individuals without back pain.

\section{Discussion}

In the present study of 40 patients (20 were patients suffering from back pain and 20 were patients who are suffering from sciatica, changes in bladder and bowel functions, numbness in the leg and decreased reflexes at the knee and ankle) were included who were referred for the MRI scans of the Lumbar spine, 
whole spine and dorso-lumbar spine where the L3, L4 and L5 vertebrae can be seen from which the FA and $A D C$ values were calculated.

The present study estimates the changes in the FA and ADC values in patients suffering from with and without lower back pain. Dallaudiere et al ${ }^{(7)}$ et al conducted a study which showed that the mean FA and Mean Diffusivity (MD) values in lumbar nerve fibres was found different in patients with any symptomatic nerve root pain and no anatomical disco radicular conflict when comparing it to the healthy volunteers. Ai-He et al ${ }^{(2)}$ conducted a study and compared FA and ADC values of compressed and noncompressed lumbar nerve fibres, the results showed that FA of compressed nerve roots were lower when compared to non-compressed nerve fibres and $A D C$ values where higher in compressed nerve roots when compared to non-compressed nerve fibres and hence the study was found similar to present study and statistical difference was found in FA and ADC values between the compressed and non-compressed lumbar nerve roots.

Eguchi $Y$ et al ${ }^{(1)}$ reported that the mean FA value were lower and mean $A D C$ values where higher in compressed nerve roots. FA values increased and ADC values decreased after surgical treatment and strong correlation was found between neurological symptoms and DTI parameters. In the present study it showed significant reduction in FA value at the side of the symptoms of the nerve roots and slight increase in ADC values.

\section{Limitations}

This study reported certain limitations namely there was no even distribution of individuals suffering from $L 3, L 4$ and $L 5$ disc compression because of which there was a significant difference in FA value in the $L 4$ region and less different in $L 3$ and $L 5$ region. DTI parameters such as FA and ADC values were not compared with the clinical data of the patients. Further studies can be carried out using larger sample size which may have better accuracy and reveal stronger conclusion.

\section{Conclusion}

In the present study six region of interest values were taken from the right and left side of L3, L4 and L5 vertebrae and the values showed a significant decrease in the FA values in the compressed nerve roots when compared to non-compressed nerve roots and increase in the ADC values.

\section{Declarations}

- Ethics approval and consent to participate-The study protocol followed was reviewed and approved by the Research Committee of Manipal College of Health Profession and Manipal Academy of Higher Education, and ethical clearance was also obtained by Kasturba Medical College and Hospital, MAHE, Manipal. A detailed explanation about the study was given by the principal 
investigator after which they provided consent for publication. All the patients included in this research gave written informed consent to publish the data contained within this study.

- Compliance with ethical standards- All ethical standards were maintained.

- Ethics reference number-IEC 379/2019

- Informed consent: A detailed explanation about the study was given by the principal investigator after which they provided consent for publication. All the patients included in this research gave written informed consent to publish the data contained within this study.

- Availability of data and material-The datasets used and/or analysed during the current study is made available by the corresponding author and attached in the supplementary files.

- Competing interests-The authors declare that they have no competing interests in this study.

- Funding-Nil

- Authors' contributions- RK conceptualized the study. RK, KN, SY and LH have given inputs in study design. $\mathrm{KN}$ collected the data. RK and KN analysed the data and wrote the first draft of manuscript and all co- authors contributed in critical review of data analysis and manuscript writing. RK will act as guarantor for this paper. All authors have read and approved the manuscript.

- Acknowledgements- Not applicable

\section{References}

1. Diffusion tensor imaging of radiculopathy in patients with lumbar disc herniation Eguchi Y., Oikawa Y., Suzuki M., Orita S., Yamauchi K., Suzuki M., Aoki Y., Watanabe A., Takahashi K., and Ohtori S. The Bone \& Joint Journal 2016 98-B:3, 387-394

2. He A, Wang WZ, Qiao PF, et al. Quantitative Evaluation of Compressed L4-5 and S1 Nerve Roots of Lumbar Disc Herniation Patients by Diffusion Tensor Imaging and Fiber Tractography. World Neurosurgery. 2018 Jul;115:e45-e52. DOI: 10.1016/j.wneu.2018.03.134

3. Balbi, V., Budzik, J., Duhamel, A. et al. Tractography of lumbar nerve roots: initial results. European Radiology 21, 1153-1159.

4. Manoliu A, Ho M, Nanz D, et al. Diffusion Tensor Imaging of Lumbar Nerve Roots: Comparison Between Fast Readout-Segmented and Selective-Excitation Acquisitions. Investigative Radiology. 2016 Aug;51(8):499-504. DOI: 10.1097/rli.0000000000000260

5. Wu, W., Liang, J., Chen, Y. et al. Microstructural changes are coincident with the improvement of clinical symptoms in surgically treated compressed nerve roots. Sci Rep 7, 44678 (2017)

6. Wang D, Wang S, Gao Y, Zhou Z, He J. Diffusion Tensor Imaging of Lumbar Vertebras in Female Adolescent Idiopathic Scoliosis: Initial Findings. Journal of Computer Assisted Tomography. 2018 Mar/Apr;42(2):317-322. DOI: 10.1097/rct.0000000000000667 
7. Dallaudière $B$, Lincot $J$, Hess $A$, et al. Clinical relevance of diffusion tensor imaging parameters in lumbar disco-radicular conflict. Diagnostic and Interventional Imaging. 2014 Jan;95(1):63-68. DOI: 10.1016/j.diii.2013.08.019.

8. Wu, W. et al. Microstructural changes are coincident with the improvement of clinical symptoms in surgically treated compressed nerve roots. Sci. Rep. 7, 44678; doi: 10.1038/srep44678 (2017).

9. Chen HB, Zhong ZW, Li CS, Bai B. Enough positive rate of paraspinal mapping and diffusion tensor imaging with levels which should be decompressed in lumbar spinal stenosis. Journal of Orthopaedic Science : Official Journal of the Japanese Orthopaedic Association. 2016 Jul;21(4):431438. DOI: 10.1016/j.jos.2016.04.008

10. Chen HB, Wan Q, Xu QF, Chen Y, Bai B. Reducing surgical levels by Para spinal mapping and diffusion tensor imaging techniques in lumbar spinal stenosis. J Orthop Surg Res. 2016;11(1):47. Published 2016 Apr 25. doi:10.1186/s13018-016-0382-1

\section{Figures}

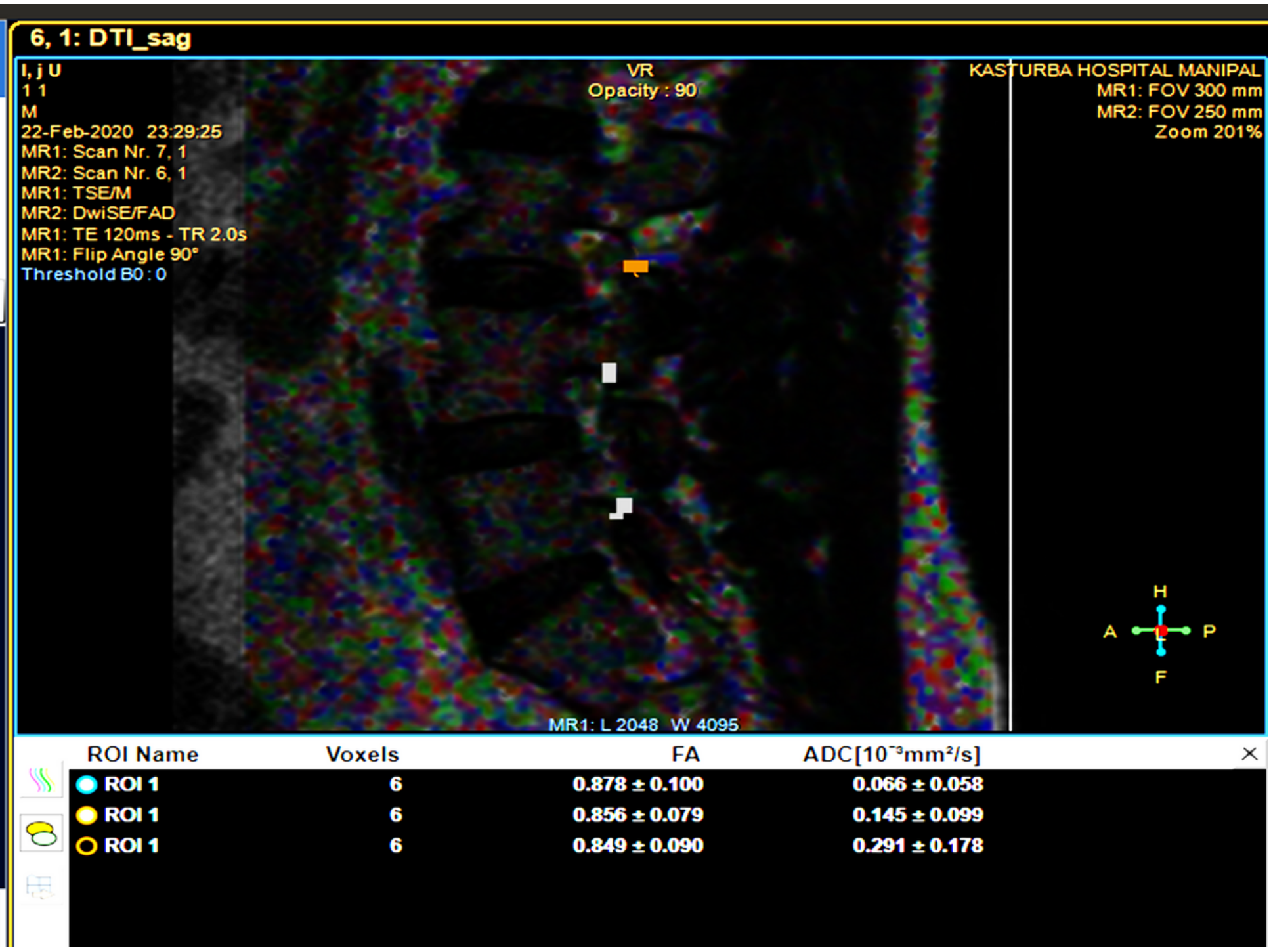




\section{Figure 1}

Shows $F A$ and $A D C$ values in right and left side of $L 3, L 4$ and $L 5$ nerve roots exiting spinal canal 